\title{
Dispersion Engineering for Multifunctional Photonic Crystal Based Nanophotonic Devices at Infrared Wavelengths
}

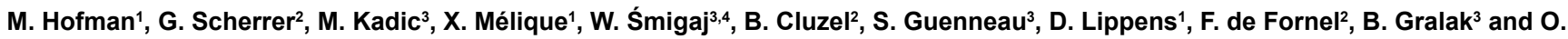 \\ Vanbésien ${ }^{1 *}$ \\ 1IEMN, UMR CNRS 8520, Université Lille 1, BP 60069, 59652 Villeneuve d'Ascq Cedex, France \\ 2OCP-ICB, UMR CNRS 6303, 9 Avenue A. Savary, BP 47870, 21078 Dijon, France \\ 3CNRS, Aix-Marseille Université, Centrale Marseille, Institut Fresnel, Marseille 13013, France \\ 4Department of Mathematics, UCL, London, UK, and Faculty of Physics, Adam Mickiewicz University, Poznań, Poland
}

\begin{abstract}
TIn this paper, we report the design, the fabrication and the near field optical microscopy of Negative Index Material (NIM) and GRadient INdex (GRIN) photonic crystal based flat lenses. They were fabricated on the basis of an InPbased photonic crystal technological platform including hole and pillar networks fabrication at nanometer scale. They show the possibility of sub-wavelength focusing by all dielectric periodic or quasi-periodic crystals. Particular attention is paid to the analysis of SNOM images using three-dimensional simulations. Finally, in order to demonstrate the versatility of our approach, a two-dimensional cloaking device mixing hole and pillar arrays is evaluated to pave the way for future integrated nanophotonic devices with complex functionalities.
\end{abstract}

OCIS codes: $220.2740,230.5298,080.2710,170.6960$

\section{Introduction}

All-dielectric artificial propagation media - including photonic crystals (PCs), meta materials and transformation optics based devices - appear as potential candidates to achieve advanced functionalities in the field of integrated nanophotonics at infrared and visible wavelengths [1-6]. The common feature of all these approaches is their dispersion engineering - and resulting ultra refraction phenomena [7-9] which occur at various wavelength scales depending on the targeted applications.

Physically, "dispersion engineering" appears as a unique tool to control with high precision wave motion in regular or graded full dielectric photonic crystals. By a proper structuring of matter at nanometer scale to operate in infrared (operating wavelength smaller than the structuring scale), we can design two dimensional refraction index maps which permit us to control light paths at will (including propagation at very short range). Such behaviors are here obtained by means of air holes etched in a dielectric medium or dielectric pillars carefully arranged on a substrate with periodic or quasi-periodic geometries fixed by the targeted optical application. The careful study of the dispersion properties (photonic band structure) of such crystals leads to unusual ultra-refraction effects (as negative refraction or self collimation for example) that can be exploited in the design of original flat lenses to focus source or plane waves with unprecedented performances. By extension, using transformation optics tools, nonperiodic designs can also be proposed to address more complex and fanciful applications such as optical invisibility.

In this paper, three paths to control light propagation and focusing at infrared $(1.55 \mu \mathrm{m})$ are explored. In each case, design (home-made code for device design and commercial FDTD software for two and three-dimensional virtual prototyping), fabrication (single mask original process using a negative resist) and characterization (scanning near field optical microscopy - SNOM - set-up) will be detailed. One main feature of our approach will be to confront, whenever possible, the accuracy of the two dimensional design processes in conjunction with the three dimensional character of fabricated devices and characterization environment to assess the "real" performances of our devices.
First, an optimized PC-based flat lens optimized in resolution $(\sim 0.8 \lambda)$ and transmission efficiency $(\sim 30 \%)$ is presented. It operates in the negative refraction regime $(\mathrm{n}=-1)$ [10-12] with a patterning scale $(\mathrm{a} / \lambda)$ of 0.3 (a denotes the crystal period). The double focusing of a quasi-point source was unambiguously experimentally assessed and validated by three dimensional (3D) simulations. Second, the focusing of a plane wave was studied by means of so-called GRIN (gradient index) lenses [13-19]. Square lattices of hole and pillar arrays were designed, operating for complementary optical field polarization, in the long wavelength regime $(\mathrm{a} / \lambda<0.1)$. Index variation in the direction transverse to the direction of propagation is obtained by varying the hole or pillar diameters while the lattice period is kept constant. Here again, the focusing is clearly evidenced by means of SNOM measurements. Then, in a prospective manner, partial invisibility $[20,21]$ was searched by means of two dimensional (2D) transformation optics concepts to go beyond initial proposals based on mixed pass- and stop- band photonic crystals [22]. Here, the idea consists in decreasing the scattering by an object deposited on a reflector $[23,24]$. A preliminary feasibility study of such a device will be initiated.

The plan of the paper is as follows: Section II is devoted to fundamental physical principles which allow us to exploit ultrarefraction phenomena using local and bulk dispersion engineering in patterned dielectrics. In section III, the InP-based technological

*Corresponding author: Olivier Vanbesien, IEMN, UMR CNRS 8520, Université Lille 1, BP 60069, 59652 Villeneuve d'Ascq Cedex, France, Tel : 0320197876 E-mail: Olivier.Vanbesien@iemn.univ-lille1.fr

Received October 23, 2013; Accepted December 21, 2013; Published Decembe 23, 2013

Citation: Hofman M, Scherrer G, Kadic M, Mélique X, Śmigaj W, et al (2013) Dispersion Engineering for Multifunctional Photonic Crystal Based Nanophotonic Devices at Infrared Wavelengths. J Nanomed Nanotechnol 4: 185. doi:10.4172/2157-7439.1000185

Copyright: (c) 2013 Hofman M, et al. This is an open-access article distributed under the terms of the Creative Commons Attribution License, which permits unrestricted use, distribution, and reproduction in any medium, provided the original author and source are credited. 
platform used to fabricate hole and pillar arrays at nanometer scale are presented. In Section IV, experimental results by means of near field optical microscopy are shown and analyzed notably by means of 3D FDTD simulations. Prospective work towards cloaking devices combining hole and pillar arrays is also given. Section $\mathrm{V}$ contains concluding remarks.

\section{Dispersion Engineering in All-Dielectric Propagation Media}

To perform dispersion engineering in all-dielectric artificial materials, two main research axes are privileged, depending on the structuring scale of the matter (a) versus operating wavelength $(\lambda)$. For a/ $\lambda$ ratios above $0.1-0.2$, we face the domain of PCs, intrinsically periodic, and whose ultra-refraction phenomena are extracted from band-structure calculations. Generally, it consists in a "bulk" dispersion engineering to reach one particular operating mode. One of the most well-known examples of this class of devices is undoubtedly the "flat lens", detailed in section 2.1 . For lower a/ $\lambda$ ratios, typically around 0.1 , we reach the effective medium (or "meta material") regime with no requisite of periodicity and, using the tools of transformation optics $[25,26]$, localized dispersion engineering becomes possible to develop

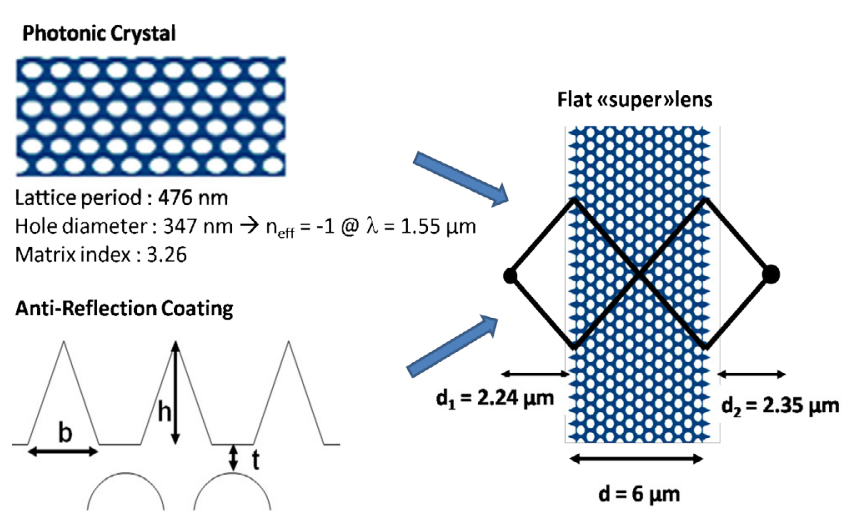

$b=238 n m-h=329 n m-t=33 n m$

Figure 1: imensioning and expected performances of the 2D PC based flat lens including anti-reflection coatings at $1.55 \mu \mathrm{m}$

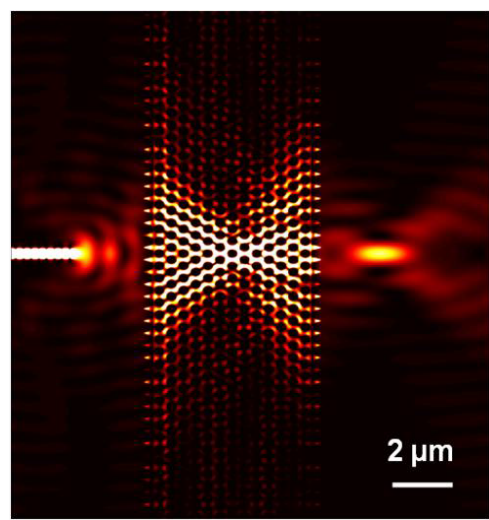

Figure 2: 2D FDTD simulation of the PC based flat lens described in Figure 1 The field amplitude for TE polarization is plotted under steady-state conditions. Light is injected from a $400 \mathrm{~nm}$ wide cut strip waveguide to mimic a "quasi" point source.
GRIN (gradient index) lenses, for example (see section 3.2) or more complex devices towards the goal of invisibility.

\section{Photonic crystal based flat lens}

Figure 1 summarizes the main characteristics of the 2D PC- based flat lens we designed. In brief, we use a 2D PC of air holes etched in a semiconductor matrix. The refractive index of the latter is set to 3.26 and corresponds to the one of the first propagating mode in an InP/ InGaAsP/InP heterostructure aimed to operate in monomode regime around $1.5 \mu \mathrm{m}$. Lattice period and air filling factor are adjusted to reach an $n=-1$ regime (crossing between the light line and a pass-band with negative slope) in the second pass-band of the PC for $a / \lambda=0.31$ for TE polarization (corresponding to a TM band gap for the given data). The triangular arrangement of air holes is used to promote isotropic behavior in terms of refraction.

As it is commonly admitted in the field of PCs, an $n=-1$ index value is not sufficient to guarantee the fabrication of a performing flat lens. Indeed, in terms of surface impedance, such an $\mathrm{n}$ value is not synonymous of $\mathrm{z}=1$, the necessary condition to match that of the adjacent air. Moreover, extraction of effective permittivity $(\varepsilon)$ and permeability $(\mu)$ which can be performed (with caution for such $a / \lambda$ values) on such crystals shows that not only $\varepsilon$ and $\mu$ are far from unity but they also vary strongly with the incidence angle of the wave impinging on the crystal. To overcome this limitation and to obtain high light transmission efficiency through the lens, anti-reflection coatings (ARCs) have been superimposed on both sides of the lens. The main concern was to ensure matching for the largest incidence angle range possible. 2D simulations of PCs covered by ARCs composed of triangles situated between two successive holes of the PC (Figure 1, middle row) yielded transmission levels higher than $80 \%$ for incidence angle as high as $60^{\circ}$.

As shown in Figure 2, which illustrates the double focusing obtained for an incident point source with a flat lens, promising performances are obtained with a very low level of reflection at the input of the lens and a well-defined and bright spot on the right hand side whose position fits merely the expected value using geometrical optics. A slight difference between $\mathrm{d}_{1}$ and $\mathrm{d}_{2}$ (theoretically perfectly equal) that is observed is probably due to the presence of the ARCs whose thicknesses are not negligible compared to the lens thickness $(10 \%)$. The estimated resolution reaches $0.41 \lambda$, which is lower than the Rayleigh limit, bringing this lens in the field of "super lenses" but it remains higher than the structuring scale as it can be expected (far from the concept of "perfect lens" that only uses effective constitutive parameters but ignores the real patterning scale).

\section{GRIN lenses}

The double focusing of a point source using a flat lens obtained by negative refraction is not the sole operating mode which could be interesting to reach using flat lenses in the field of nanophotonics. Even if the previous lens possesses interesting properties in terms of applications, notably the fact that it behaves very differently versus the nature of the incident wave (fully transparent in theory for a plane wave and focusing for a point source), the opposite behavior could also be of interest: the focusing of a plane wave. GRIN lenses, for GRadient INdex lenses, have been discovered a long time ago but very few have been engineered up to now for integrated nanophotonics. In this area, our ability to engineer dispersion using periodic or pseudo-periodic PCs or meta materials can be helpful to design original and efficient lenses?

Figure 3 illustrates an approach that can be developed based by 


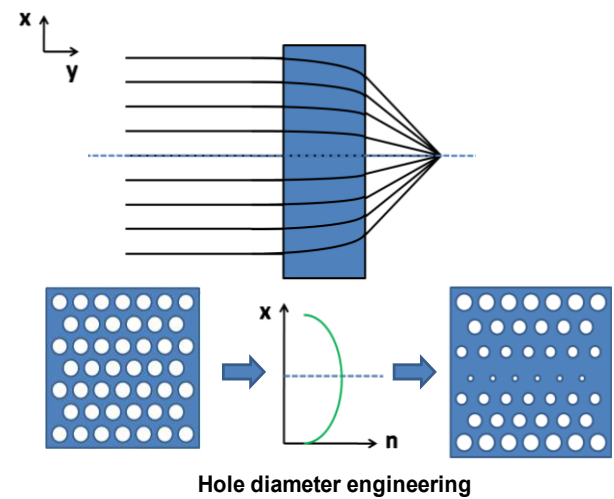

Figure 3: GRIN lens design by local hole diameter engineering of a 2D photonic crystal to mimic the parabolic profile of the refractive index (effective medium approach). Note that the variation of profile is only along the $x$-axis, hence the PC periodicity along y.

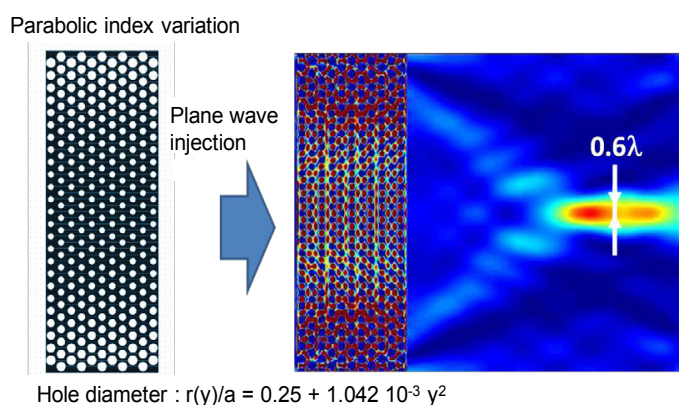

Figure 4: 2D FDTD simulation of the focusing of a plane wave by a PC based GRIN lens at $1.55 \mu \mathrm{m}$. The field amplitude for TE polarization is plotted under steady-state conditions (hole array etched in the semiconductor matrix).

changing some of the parameters of a PC lattice to modify locally its effective refractive index. Let us mention here that what matters to control precisely the propagation of light in the device is the index difference between two regions of space. The origin of this gradient, i.e. whether it arises from a domain with positive or negative index values, is not fundamental. This explains why such gradients can be obtained for low a/ $\lambda$ values by exploiting for example the slope of the first band of an all-dielectric PC, slope whose amplitude depends mainly on two coupled parameters: the lattice period and the filling factor. Moreover, as a local engineering is needed and has to operate for a particular incidence angle, most of the constraints developed in the previous paragraph concerning isotropy for impedance matching are here relaxed. Additional degrees of freedom will exist for the design of GRIN lens, such as the use of hole or pillar arrays, as shown in the following.

Since, the effective index in the first band of a PC is a direct consequence of the filling factor, it appears natural, for a fixed lattice period, to diminish the air hole diameter (Figure 3) or to increase the semiconducting pillar diameter if one wants to increase the local index. Also, it can be shown that a parabolic variation of the index in the direction normal to the direction of the propagation makes optical rays bend towards a specific point of the real space.

Figure 4 shows a 2D FDTD simulation of a GRIN lens based on a hole diameter engineering at $1.55 \mu \mathrm{m}$ starting from a PC equivalent to the one described for the flat lens. A $0.6 \lambda$ spot size is obtained. Here, given that geometrical changes are required, a crucial issue is to estimate the range of particle, hole or pillar, diameters within reach of our technological platform.

\section{Technological Platform}

The all-dielectric prototypes developed in the following make use of a common one-mask technological process. It allows us to create the different building blocks including the devices and their environment: optical waveguide for injecting and/or collecting light as well as hole or pillar arrays for the active zones.

To build the $2 \mathrm{D} 1 / 2$ photonic devices, we start from an InP-based heterostructure grown by molecular beam epitaxy: Superstrate InP $(0.2$ $\mu \mathrm{m})$ / Core In GaAsP $(0.5 \mu \mathrm{m}) / \mathrm{InP}(1.3 \mu \mathrm{m})$ / InP Substrate. Based on such a heterostructure, it is possible to confine light in a plane (in the direction of growth) and to use a unique propagation mode. This propagation can then be modulated in the other two spatial directions by a $2 \mathrm{D}-\mathrm{PC}$. The transverse propagation mode is characterized by an effective index which is found to be 3.26 .

Fabrication of PCs for near-field and infrared operation, or even towards the visible range, relies on exploiting the most advanced microand nanotechnologies. These are electron lithography and deep etching, respectively. The first allows us to define the pattern and geometry according to the nanometric scale, while the second allows us to make holes as well as define pillars with a high aspect ratio and anisotropy. The technological process that we will describe briefly draws its originality from its simplicity, that is to say a unique mask allowing us to define large plots and/or guides (several dozen or hundreds of $\mu \mathrm{m}$ long) and the lattice of sub-micronic holes in a single step. This same mask is used also for etching. Figure $5 \mathrm{a}$ details the process developed while Figure $5 \mathrm{~b}$ illustrates some results obtained at different stages of the process.

The technological process relies on the use of a negative electronic resist, hydrogen silsesquioxane (HSQ), which is well known for its resolution $(\sim 3-8 \mathrm{~nm})$ and its capacity to resist plasma attacks after oxidation. Moreover, its negativity implies that only written zones will remain in place after development. This represents an undeniable advantage for our structures, insofar as the optical wave guides (narrow at $0.4-0.6 \mu \mathrm{m}$, but long at some $\mathrm{mm}$ ) and the PC (with hole filling factor approaching 50\%) can be simultaneously defined.

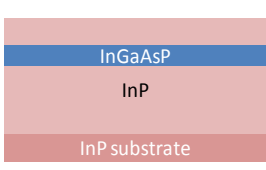

(a) InP based heterostructure

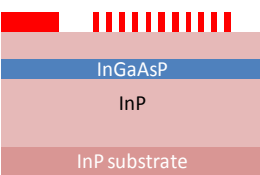

(d) $\mathrm{KOH}$ development

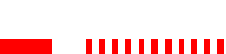

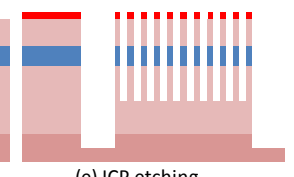

(e) ICP etching after HSQ oxidation

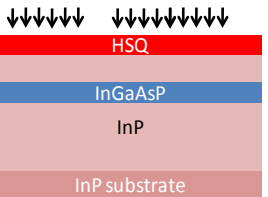

(c) e-beam lithography

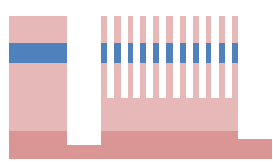

(f) cleaning process
Figure 5a: Side views of the technological steps of the one-mask process based on a III-V InP based heterostructure. 


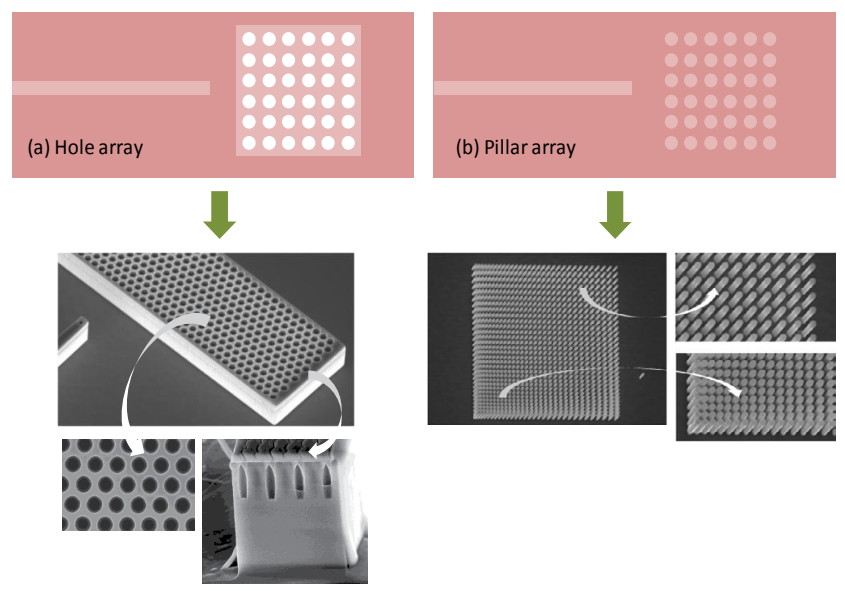

Figure 5b: Schematic top views of typical fabricated photonic crystals using hole (a) or pillar (b) arrays and optical waveguide for operation at $1.55 \mu \mathrm{m}$. Typical dimensions (lattice period, hole or pillar diameter) ranges from 100 to $400 \mathrm{~nm}$ and SEM view of fabricated devices.
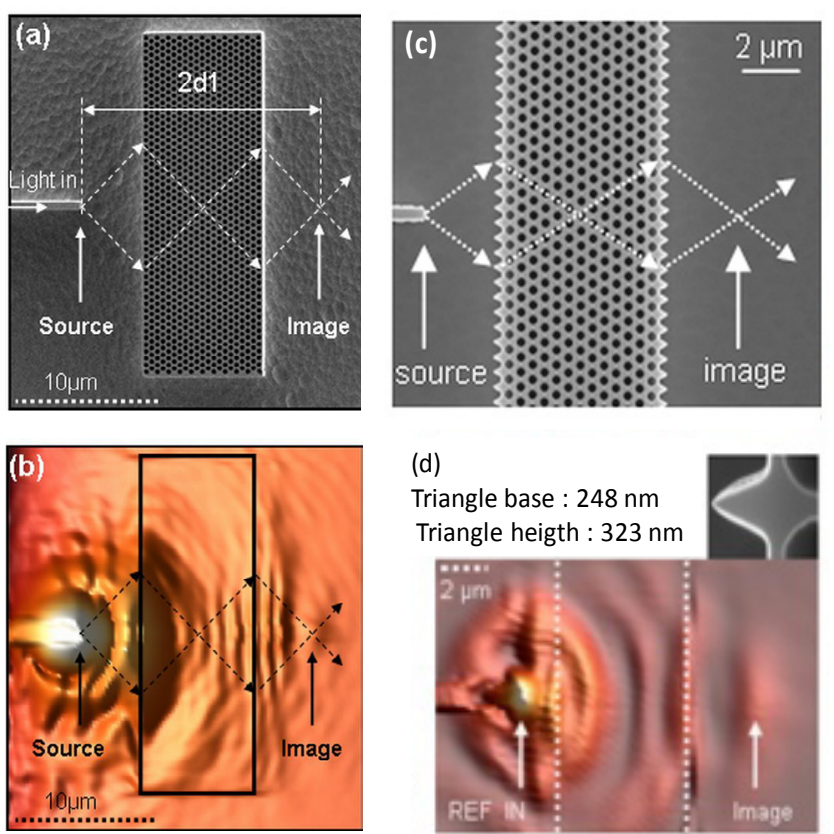

Figure 6: Double focusing experiments using $P C$ based $n=-1$ flat lenses at $1.55 \mu \mathrm{m}$ defined in Figure 1(a) and (b) : SEM view and SNOM image of the bare device (21 rows). (c) and (d) SEM view and SNOM image including antireflection coatings (13 rows)

In short, a resist thickness of approximately $500 \mathrm{~nm}$ is deposited on the epitaxial layer. After looking for an optimal dose and fine correction of the proximity effects, a stage of electronic lithography allows us to describe the pattern to be created in the resist. This pattern integrates rectangular structures with a high length/width aspect ratio and discs that are sub-micronic in size but high in density. Non-exposed resist is dissolved with a $\mathrm{KOH}$ developer. The remaining resist is then oxidized and acquires a hardness that is sufficient to withstand etching of the semiconductor epitaxial layer. Etching is done by inductive coupled plasma, with a gas of the type $\mathrm{Cl}_{2} / \mathrm{H}_{2} / \mathrm{CH}_{4}$, allowing us to obtain the desired anisotropic etching with the high aspect ratio after optimization.
Width/diameter ratios for the holes of approximately five to 10 can be obtained, allowing us to reach the $2 \mathrm{D}^{1 / 2} / 2$ regime we are looking for. Figure $5 \mathrm{~b}$ illustrates some results obtained showing the large regularity obtained for the lattice associated with the perfectly defined etching flank for the lens. The same approach is used to fabricate the ARCs on both sides of the lens or for the fabrication of pillar arrays. In this last case, special attention is paid to the deep etching since its dynamic differs (species evacuation) compared to the hole formation.

\section{Experiments and Analysis}

\section{SNOM measurements}

Figure 6 shows SNOM (scanning near filed optical microscope) measurements which illustrates the double focusing effect with an $\mathrm{n}=$ -1 PC based flat lenses at $\lambda=1.55 \mu \mathrm{m}$. In the first case (a) and (b), which corresponds to a bare 21-rows lens, a focused spot with a low intensity, approximately $1 / 100^{\mathrm{e}}$ of the incident power, is registered at the expected position behind the lens (about $4.3 \mu \mathrm{m} /$ half the lens thickness for an equivalent source-lens distance). The associated resolution, taking into account the finite dimension of the probe is estimated to $0.8 \lambda$. The second set of experiments (c) and (d) tests the behavior of 13-row lenses covered by ARCs. Several different ARC designs (triangle bases and heights varying from $200 \mathrm{~nm}$ to $250 \mathrm{~nm}$ and from $290 \mathrm{~nm}$ to 325 $\mathrm{nm}$ respectively) were used to test the robustness of our theoretical approach to technological constraints. In all cases, the transmission light efficiency is significantly increased compared to the bare lens to reach a maximum estimated about $30 \%$. In this last case, resolution is close to $\lambda$, probably due to a set of PCs with higher propagation losses due to less deep holes obtained during the etching process.

Such a result is however extremely encouraging, even if from the $2 \mathrm{D}$ design process to the $3 \mathrm{D}$ prototype reality, many parameters can cause a slight shift of the optimal operating wavelength around the targeted value (around a few per cent). Moreover, the ARC design which tolerates a large range of incidence angles remains resonant in wavelength and is also designed for a precise wavelength. Here also, small deviations from the initial mask design to the fabricated lens interface can be obtained. This quite general issue of $3 \mathrm{D}$ reality versus $2 \mathrm{D}$ design will be addressed in the next paragraph.

Figure 7 illustrates the preliminary results obtained in the case of hole-based or pillar-based GRIN lenses also at $1.55 \mu \mathrm{m}$. As a major difference compared to flat focusing lenses, one can observe the injection region which consists of a large (three times the lens dimension) aperture cut waveguide to generate a quasi-plane wave. In practice we start from a classical optical waveguide, as before, but instead of keeping it at a sub-wavelength dimension up to the vicinity of the lens, this "horn" waveguide is progressively widened, over a distance much longer than the guided wavelength to avoid extra losses. As seen on the SNOM images, it allows us to define a planar wave front as expected. For the two lenses, a focus spot is obtained at the output of the lens at the position anticipated by the FDTD calculations $(0.7 \lambda)$. In the hole case, the width of this spot is about $0.8 \lambda$ (taking into account the probe finite dimension), close to the expected theoretical value. In the pillar case, a larger spot is unexpectedly obtained which remains to be fully interpreted.

\section{Three dimensional analysis}

In the field of nanophotonics making use of PCs and/or metamaterials to exploit abnormal refraction properties, most of the designing procedures are carried on $2 \mathrm{D}$ models. By essence, reality is intrinsically $3 \mathrm{D}$ and so are the fabricated prototypes. The analysis of 


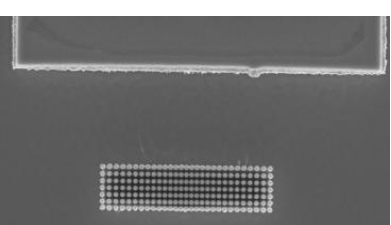

pillar array

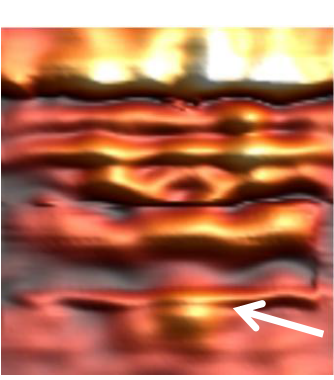

incident plane wave
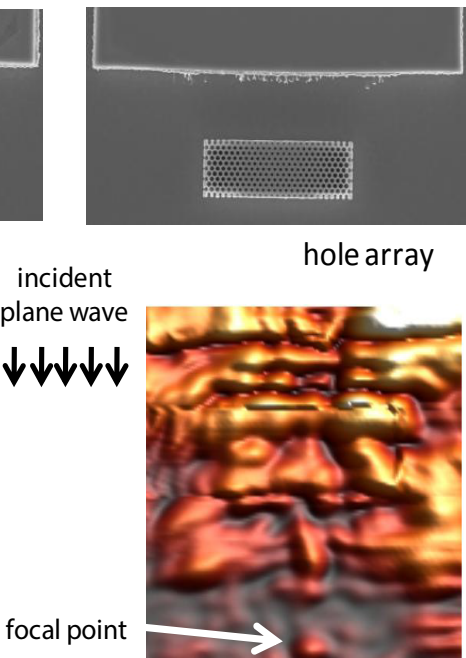

hole array

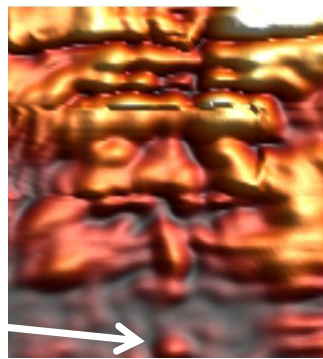

Figure 7: SEM views and SNOM images for the focusing of a plane wave by a pillar-based (left side) or hole-based (right side) GRIN lens at $1.55 \mu \mathrm{m}$.

3D Simulation : Image at a given height (1.6 $\mu \mathrm{m}$ above the substrate)

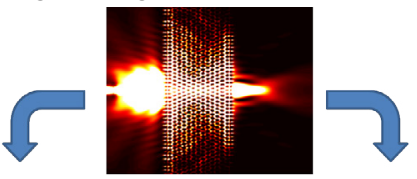

3D Simulation : Image reconstruction following the SNOM probe course
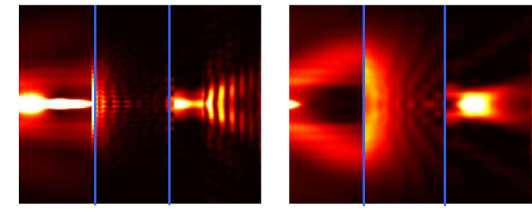

A few nm above the substrate

$1.6 \mu \mathrm{m}$ above the substrate

SNOM measurements The SNOM probe follows the device topology (variable height)
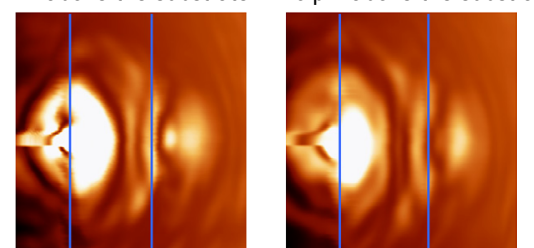

Figure 8: Analysis of SNOM images using 3D FDTD simulation of the double focusing experiment by a flat lens.

measurements becomes thus sometimes difficult when exclusively compared to 2D simulations. Erroneous conclusions can even be drawn from a careful study of experimental images.

To avoid such misguided interpretations, 3D calculations are required even if they may be impractical for optimization purposes due to the calculation time and memory space requirements needed to obtain sufficient precision. However, for reverse engineering, a post$2 \mathrm{D}$ numerical image reconstruction is particularly information-rich. Figure 8 illustrates such an approach dedicated to the double focusing experiment by an $n=-1$ flat lens evaluated using SNOM images.

In practice, a SNOM image is formed by recording the optical power coupled to a probe which follows the device topology at a given distance, and thus at a variable absolute altitude. Looking at Figure 5b, this means that the reconstructed image is composed of information collected at different heights and does not correspond to the case of a 2D simulation which presupposes invariance in the transverse direction. To obtain simulated images as close as possible to the experimental ones, shown at the bottom of Figure 8, we start from a full 3D FDTD device simulation (taking into account the epitaxial sequence in the vertical direction, the input waveguide, the real lens height and finite depth of holes, ARCs, ...) and extract the calculated optical field using the topology map given by the SNOM. Such a procedure has been applied for two distinct SNOM probe courses: a few nanometers (left side of figure 8 ) and $1.6 \mu \mathrm{m}$ (right side of Figure 8) above the surface. Behind the lens this latter distance corresponds to the longitudinal plane of the quantum well of the confining heterostructure and thus to the plane where maximum optical power is expected.

Strong similarities between SNOM and such reconstructed images can be evidenced. One of the main added values of the approach concerns the spot size estimation. It is very tempting using SNOM images to claim a sub-wavelength resolution using the image corresponding to a probe course close to the substrate (left side of Figure $8)$. However in this case, the spot position is too close from the output interface of the lens compared to the targeted operating regime, and do not respect Snell's law of refraction for $\mathrm{n}=-1$. On the contrary, the right hand side images, calculated in the quantum well plane of the confining heterostructure, give larger spots, not sub-wavelength (between $0.8 \lambda$ and $\lambda$ ), but correctly positioned. This shows the extreme difficulty of interpreting SNOM images directly, especially when devices have rough surfaces and a probe which follows their topology at a fixed distance.

Despite these necessary precautions, our 3D analysis has unambiguously confirmed that the measured phenomena were those expected and optimized using a 2D approach. Excellent performances have been obtained both for flat lenses and GRIN lenses at optical wavelengths. However, the 2D picture of a double focusing using a PC based flat lens or plane wave focusing using a GRIN lens is often too simple to evaluate resolutions and a fully $3 \mathrm{D}$ approach is required to predict the real performance of such devices for applications in future integrated photonic circuits.

\section{Concluding Remarks and Prospects}

At this stage, we have shown the ability of full dielectric periodic or pseudo-periodic $2 \mathrm{D}^{1} / 2$ crystals to reach interesting performances at infrared wavelengths (around $1.55 \mu \mathrm{m}$ ) as focusing lenses (for point sources or plane waves) using bulk or localized dispersion engineering.

Concerning negative refraction based flat lenses for point source focusing; high transmission efficiency (of about 30\%) has been experimentally obtained with lens resolution in the range of 0.8 to 1 $\lambda$. These state of the art results for photonic crystal based devices are supported by a challenging full three dimensional calculation which permits a complete analysis of the double focusing process in its characterization environment (here near field optical microscopy).

For plane wave focusing using gradient index based flat lenses, we have also shown that, on a practical side, both hole and pillar based approaches for patterning a semiconducting heterostructure can be employed with similar performances in terms of lateral resolution $(0.7$ to $0.8 \lambda$ ) and transmission efficiencies.

If it is commonly accepted now that PC based devices will not reach outstanding performances in terms of sub-wavelength resolution for example due to their structuring scale compared to the promises of metamaterials, they are undoubtedly the most mature systems for 
Citation: Hofman M, Scherrer G, Kadic M, Mélique X, Śmigaj W, et al. (2013) Dispersion Engineering for Multifunctional Photonic Crystal Based Nanophotonic Devices at Infrared Wavelengths. J Nanomed Nanotechnol 4: 185. doi:10.4172/2157-7439.1000185

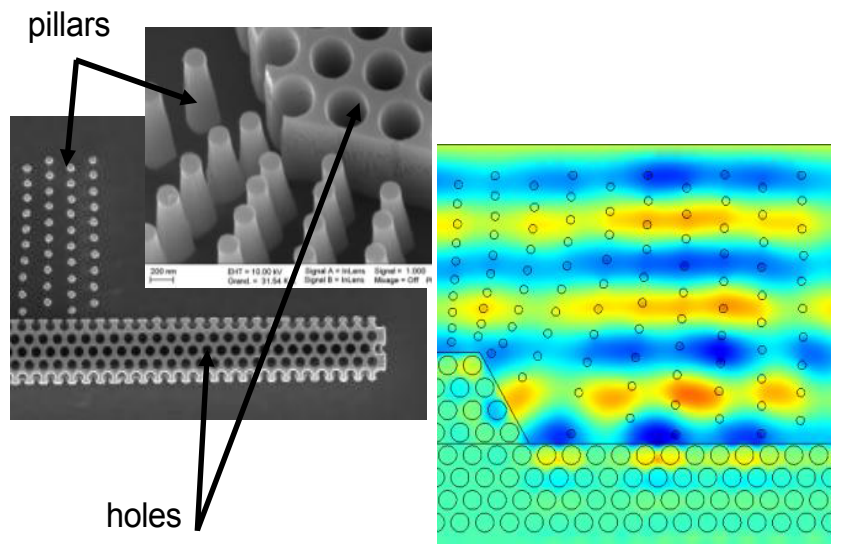

Figure 9: Towards transformation optics based devices: combination of holes and pillars in a single technological process (left) for a metamaterial which mimics a flat mirror, as suggested by the nearly flat wavefront of the total magnetic field phase computed in TE polarization (right).

applications at optical wavelengths. To deepen our knowledge, the next development stages of such devices can be envisaged in two main directions.

First, can the field of transformation optics rich of proposals for manipulating light at ultra-short scale be explored using all-dielectric structures $[27,28]$ ? One possible avenue would be to design geometries mixing holes and pillars. As shown in Figure 9, our technological "single mask" process can fully meet these objectives. In this case, the main practical challenge is to evaluate the ultimate limits in dimensions for pillar or hole diameters and heights we are able to fabricate to fulfill the stringent precision of dispersion required by the theory of transformation optics to perform cloaking or routing functions at optical wavelengths. More details can be found in [29].

The second research axis consists in understanding how the fabricated lenses could be of use for more complex applications for detection and/or imaging [30]. Preliminary results for infrared detection and imaging, inspired by tomography in reflection, including an $\mathrm{n}=-1$ flat lens have been proposed [31]. These works are currently pursued with GRIN lenses in a transmissive approach.

In all cases, it is believed that the performances reached by alldielectric devices at optical wavelengths open right now the way for future innovative applications for integrated nanophotonics.

\section{Acknowledgement}

This work was founded by Agence Nationale de la Recherche under contract PNANO - FANI.

\section{References}

1. Pendry JB (2000) Negative refraction makes a perfect lens. Phys Rev Lett 85 3966-3969.

2. Pendry JB, Schurig D, Smith DR (2006) Controlling electromagnetic fields. Science 312: 1780-82.

3. Shalaev MV, Cai W, Chettiar UK, Yuan HK, Sarychev AK, et al. (2005) Negative index of refraction in optical metamaterials. Optics Letters 30: 3356-3358.

4. Kosaka H, Kawashima T, Tomita A, Notomi M, Tamamura T, et al. (1998) Superprism phenomena in photonic crystals. Phys Rev B $58: 10096$.

5. Engheta N, Ziolkowski RW (2005) A positive future for double negative metamaterials. IEEE Transactions on MTT 53: 1535-1556.
6. Arlandis J, Centeno E, Pollès R, Moreau A Campos J, et al. (2012) Mesoscopic Self-Collimation and Slow Light in All-Positive Index Layered Photonic Crystals Physical Review Letters, 108: 037401.

7. Dowling JP, Bowden CM (1994) Anomalous index of refraction in phtotonic bandgap materials. J. Mod. Optics 41: 345-351.

8. Enoch S, Tayeb G, Sabourous P, Guerin N, Vincent P (2002) A metamaterial for directive emission. Phys. Rev. Lett. $89: 213902$.

9. Baba T, Abe H, Asatsuma T, Matsumoto T (2010) Photonic crystal negative refractive optics". Journal of Nanoscience and Nanotechnology 10: 1-9.

10. Fabre N, Lalouat L, Cluzel B, Mélique X, Lippens D, et al. (2008) Optical nearfield microscopy of light focusing through a photonic crystal flat lens. Phys. Rev. Lett 101: 073901.

11. Smigaj W, Gralak B, Pierre R, Tayeb G (2009) Antireflection gratings for a photonic crystal flat lens. Optics Letters 34: 3532-3534.

12. Scherrer G, Hofman M, Smigaj W, Gralak B, Mélique X, et al. (2010) Interface engineering for improved light transmission through photonic crystal lenses. Appl. Phys. Lett 97: 071119.

13. Wu Q, Gibbons JM, Park W (2008) Graded negative index lens by photonic crystals. Optics express 16: 16941

14. Gaufillet F, Akmansoy E (2012) Graded photonic crystals for graded index lens Optics Communications 285: 2638-2641.

15. Liu M, Yun M, Xia F, Kong W, Lv W, et al. (2013) Superlens realized by the two dimensional graded photonic crystal. Optik - International Journal for Light and Electron Optics 124: 1-3.

16. Tan C, Niemi T, Peeng C, Pessa M (2011) Focusing effect of a graded index photonic crystal lens. Optics Communications 284: 3140-3143.

17. Cassan E, Do KV, Dellinger J, Roux XL, de Fornel F, et al. (2013) Polarization beam splitting using a birefringent graded photonic crystal. Optics Letters 38 : 459-461.

18. Dyachenko PN, Pavelyev VS, Soifer VA (2012) Graded photonic quasicrystals Optics Letters 37: 2178-2180.

19. Chiang TM, Guenneau S, Hazard J, Enoch S (2011) Focusing light through a stack of toroïdal channels in PMMA. Optics Express 19: 16154-16159.

20. Zhang B, Luo Y, Liu X, Barbastathis G (2011) Macroscopic invisibility cloak fo visible light. Physical Review Letters 106: 033901.

21. Gharghi M, Gladden C, Zentgraf T, Liu Y, Yin X, et al. (2011) A carpet cloak for Visible light. Nanoletters 1: 2825-2811.

22. Vanbésien O, Fabre N, Mélique X, Lippens D (2008) Photonic crystal based cloaking device at optical wavelengths. Applied Optics 47: 1358-1362.

23. Renger J, Kadic M, Dupont G, Acimovic SS, Guenneau S, et al. (2010) Hidden progress: broadband plasmonic invisibility. Optics Express 18: 15757-15768.

24. Li L, Pendry JB (2008) Hiding under the carpet: A new strategy for cloaking Phys. Rev. Lett 101: 203901.

25. Ward AJ, Pendry JB (1996) Refraction and geometry in Maxwell's equations J. Mod. Opt. 43: 773-793.

26. Nicolet A, Remacle JF, Meys B, Genon A, Legros W (1994) Transformation methods in computational electromagnetic. J. Appl. Phys $75: 6036-6038$.

27. Schurig D, Mock JJ, Justice BJ, Cummer SA, Pendry JB, et al. (2006) Metamaterial electromagnetic cloak at microwave frequencies. Science 314 977-9780.

28. Centeno E, Cassagne D (2005) Graded Photonic crystals. Optics Letters 30 2278-2281.

29. Scherrer G, Hofman H, Smigaj W, Kadic M, Chang TM, et al. (2013) Photonic crystal carpet: Manipulating wave fronts in the near field at $1.55 \mu \mathrm{m}$. Phys. Rev. B 88: 115110 .

30. Wang G, Fang J, Dong XT (2007) Refocusing of backscattered microwaves in target detection by using LHM flat lens. Optics Express 15: 3312.

31. Hofman M, Lippens D, Vanbésien O (2010) Image reconstruction using photonic crystal based flat lens operating at $1.55 \mu \mathrm{m}$. Applied Optics 49:5806 5813. 